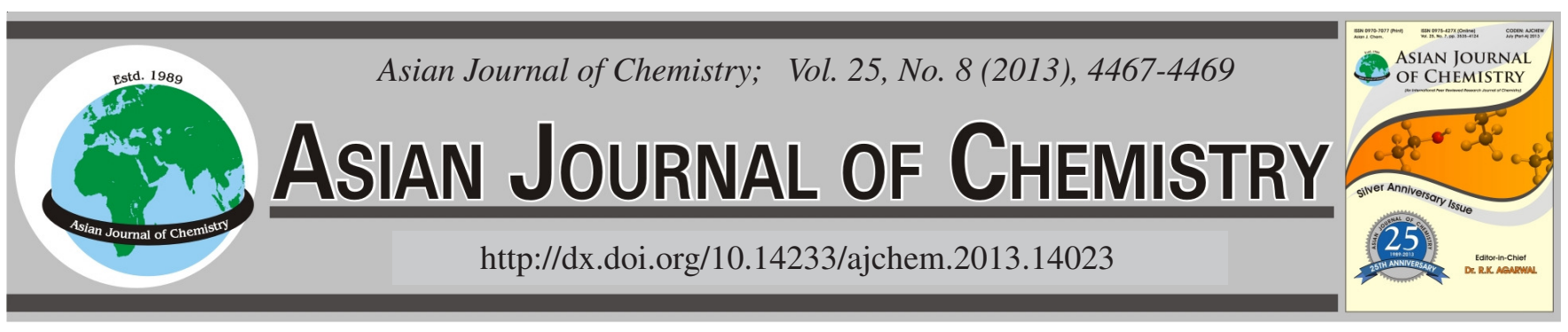

\title{
Silver Mirror Reaction with Polystyrene Nanoparticles Template
}

\author{
Fu Jun Yin ${ }^{1, *}$, Li Hong Zhang ${ }^{2}$, Hong ZhaO ${ }^{2}$, Gui ZhaO ${ }^{2}$ and XING You Xu ${ }^{3}$
}

\begin{abstract}
${ }^{1}$ Jiangsu Marine Resources Development Research Institute of Huaihai Institute of Technology, Lian Yun Gang 222005, P.R. China ${ }^{2}$ Department of Chemical Engineering, Huaihai Institute of Technology, Lianyungang 2220052, P.R. China

${ }^{3}$ Huaiyin Insititute of Technology, Huaiyin 223003, P.R. China
\end{abstract}

*Corresponding author: Fax: +86 518 85863269; Tel: +86 518 85863269; E-mail: yinfujun2008@126.com

(Received: 7 May 2012;

Accepted: 13 February 2013)

AJC-12990

Two-dimensional silver nanocavity array was fabricated by templating of the periodically assembled polystyrene nanoparticles via silver
mirror reaction. We reveal the mechanism of silver growth in the space among the polystyrene nanoparticle. Polystyrene is made of
emulsifier-free emulsion polymerization. The silver grow does not depend on specific position on the polystyrene nanoparticle surface, as
homogeneous distribution of the Ag core, relying on active surface on $\mathrm{Ag}$ core and the left channels, silver grow from silver nanoparticls
to two-dimensional silver nanocavity array.
Key Words: Nanocavity array, Polystyrene nanoparticle, Self-assembly, Template.

\section{INTRODUCTION}

Bottom-up fabrications of metals into two- and threedimensional nano-architectures have recently stimulated great interest of the researchers in many fields and inspired the development of various nanodevices for future technical optical and electrical applications ${ }^{1-5}$. By creating metal nanostructures, it is possible to control fundamental properties of metals, this should consequently enable us to develop new materials and advanced devices of desirable properties and functions. The diverse optical properties of metal nanoparticles due to the excitation of the localized surface of the nanosized metal structures have been widely investigated and applied in the studies of many fields ${ }^{6-11}$.

Motivated by various potential applications of metallic nanocavity arrays, the fabrications of the ordered metallic nanocavity arrays have recently been an attractive and challenging subject. Using assembled periodic structures of monodispersive particles to create the metallic nanocavity array has proved to be a flexible and reliable approach. Jiang ${ }^{12}$ prepared the two-dimensional ordered void arrays of metals and semiconductors by physical vapor deposition techniques on the assembled colloidal crystals of silica and polystyrene nanoparticles. Photonic metal films were prepared by electrochemical reduction of metal complex ions within the interstitial spaces between polystyrene latex spheres self-assembled on a gold-coated surface ${ }^{13}$.

Herein, we demonstrate a simple method to prepare silver nanocavity array by chemical deposition of silver metal on an ordered periodic structure of polystyrene submicrometer colloid particles.

\section{EXPERIMENTAL}

4-Amino thiophenol (PATP) was purchased from Acros Organics Chemical Co. and used without further purification. The other chemicals are all reagent grade.

Apparatus and methods: Monodispersive polystyrene nanoparticles were synthesized by emulsifier-free emulsion polymerization method ${ }^{14}$. The size of the particles estimated by the TEM measurement was $c a .520 \mathrm{~nm}$. Fig. 1 is the sketch to form silver nanocavity array. The highly ordered template of the polystyrene nanoparticles was fabricated by selfassembly technique: A glass slide was set vertically in a polystyrene colloid, the film of the highly ordered polystyrene nanoparticles was obtained on the slide as the solvent of the colloid was evaporated at room temperature. Chemical deposition of silver was conducted by dispersing a freshly mixed solution of $0.05 \mathrm{M} \mathrm{AgNO}_{3}, 0.5 \mathrm{M}$ glucose and small amount of ammonia onto the surface of the polystyrene nanoparticle template. The reaction was carried out at room temperature for $3 \mathrm{~h}$. Then the remaining reaction solution was completely ultrapure water. The resulting silver film was peeled off by a tape and immersed into a toluene solution to dissolve the polystyrene nanoparticles. After that, the nanocavity array structure of silver was obtained. The surface morphologies of the samples were measured on a Hitachi 7350 G SEM microscope. 


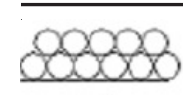

(a)

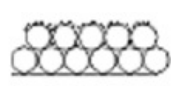

(b)

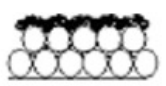

(c)

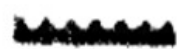

(d)

Fig. 1. (a) Ordered assembly of polystyrene nanoparticles as the template (b) Chemical deposition of Ag metal for few minutes (c) Chemical deposition of Ag metal for half hour (d) Removal of polystyrene particles

\section{RESULTS AND DISCUSSION}

Once the ordered opal is formed, it can be infiltrated with nanosized crystallites or a precursor of insulators, semiconductors or metals ${ }^{15,16}$. The bottom-up deposition of Ag have been realized by the electron beam deposition (EBD) ${ }^{7}$. The chemical bath deposition (CBD) method ${ }^{17}$ is adopted to upbottom deposition Ag. Silver-mirror reaction has been widely employed in the efficient coating of silver metallic thin film on substrates such as glass. Similarly, we prepared the silver nanocavity array on the ordered structure of polystyrene nanoparticles via the reduction reaction of silver ions with glucose. Silver film was chemically generated on the top of polystyrene nanoparticles. The metallic film with the templating polystyrene nanoparticles can be easily peeled off. The silver nanocavity array structure was then obtained by removing the polymer particles with toluene. The SEM images of the assembled polystyrene nanoparticles and silver nanocavity array are shown in Fig. 2. The assembly of polystyrene nanoparticles by solvent evaporation resulted in the formation of a close-packed crystal structure (Fig. 2a). The array structure of the polystyrene nanoparticles was then used as the template for the deposition of silver. silver film was peeled off by a adhesive tape without removing the template (Fig. 2b). It is inverse of the template. From Fig. 2b, it can be clearly seen that the silver had filled into the space among the polystyrene nanoparticle. There are also some adhered polystyrene nanoparticles outside the silver film. It is reasonable that the dense metallic film could block the diffusion of the reactants into the deeper layers of the polystyrene nanoparticles. Consequently, the remaining reactants inside the template gradually exhausted and this limited amount of reactants may only result in a slight increase of the amount of the deposited silver metal inside. As a result, the silver metal at the template side mainly retains its original semi-spherical cavity shape at the superficial layer of the polystyrene nanoparticles. Dissolve the template with toluene, we obtain the silver nanocavity array structure (Fig. 2c), nanocavities match the size of the polystyrene nanoparticles and the array retains the two-dimensional close-packed ordering. At the bottom of the nanocavity, no holes as those observed in threedimensional inverse opal ${ }^{8}$, were observed in this case, indicating that only the top layer of the polystyrene nanoparticles was involved in templating the formation of the silver nanocavity array. Furthermore, the repeated experiments demonstrated that only semi-spherical nanocavities could be obtained under our experimental conditions. In the same way, silver mirror reaction on clean glass without polystyrene nanoparticles template is out-of-order (Fig. 2c), although it is luminant like a mirror. The contrast from Fig. 2c and Fig. 2d, we conclude that silver grow in the determinative space and random deposit is completely different.
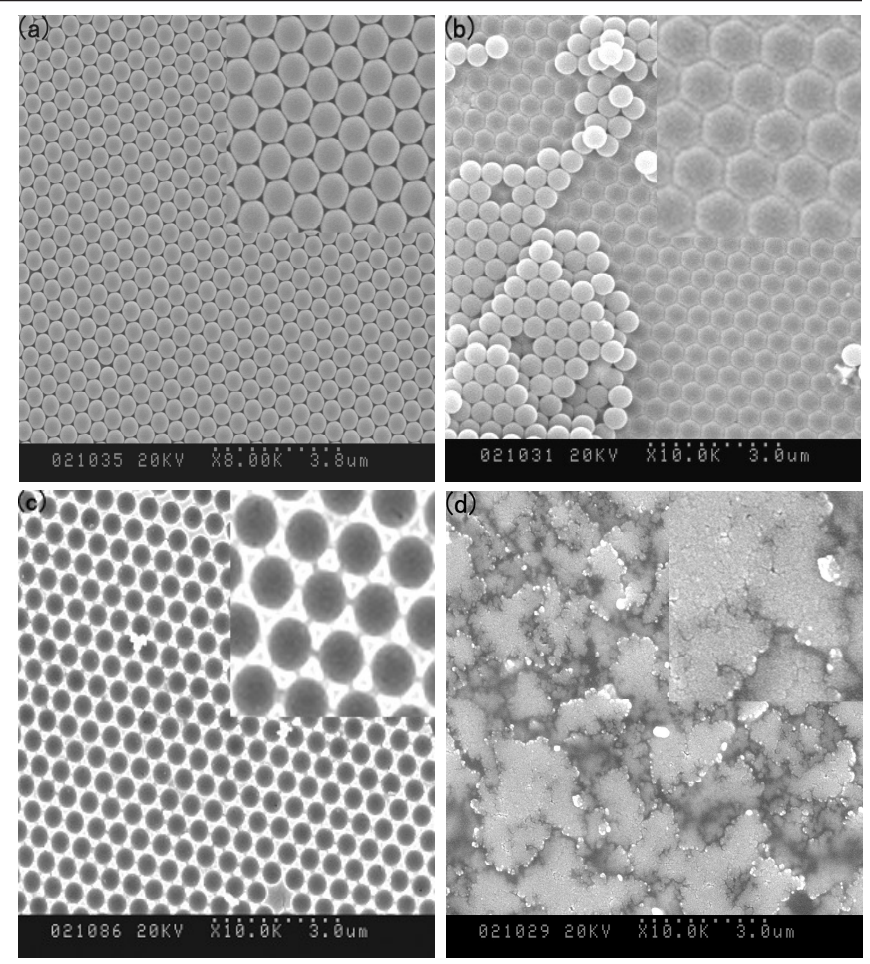

Fig. 2. SEM graphs (a) polystylene templates (b) backside of deposited silver with template; (c) after removal of template; (d) silver film formed without template

To penetrate the formation of the semi-spherical nanocavities, the morphologies of the deposited silver were tracked at different reaction stages, the results have been shown in Fig. 3. It is apparent that silver nanoparticles whose diameter is $c a .50 \mathrm{~nm}$ in $5 \mathrm{~min}$ were formed and filled into the interspace of the orderly arranged polystyrene spheres (Fig. 3a). As the reaction continues, the small metal particles congregate together and grow into the large particles (Fig. 3b), although there are interspaces left, which allow reactants continuously come into. At the same time, the larger silver nanoparticles grow up and the tighter array structure can be got. The morphology of the silver film at the polystyrene side demonstrates the deposited silver metal film maintains its ordered array structure (Fig. 3c). Within $1 \mathrm{~h}$, the reactants used up, silver fill the nanochannels of the opal uncompletely, a few of nanoparticles can be seen from observation of SEM. In order to ensure a larger filling ratio of silver amine solution in the interstitial space of the colloidal crystals, We place the samples (Fig. 3c) into fresh reactants again, obtain the samples (Fig. 3d). Eventually, a dense film of silver metal was formed on the superficial layer of the polystyrene nanoparticles template. each nanocavity is highly ordered, separated from each other and free-standing half sphere, forming a two-dimensional nanocell array. The cell is interconnected by six tiny tunnel at the surface, which is actually the contact point among the polystyrene spheres on the same dimension. It should be pointed out that polystyrene is made of emulsifier-free emulsion polymerization, Ag growth does not seem to depend on either opal order or specific position on the sphere surface, as homogeneous distribution of the Ag core. Instead of small grains fuse together to form the three-dimensionally ordered macroporous solid ${ }^{18,19}$, these samples are two-dimensional 


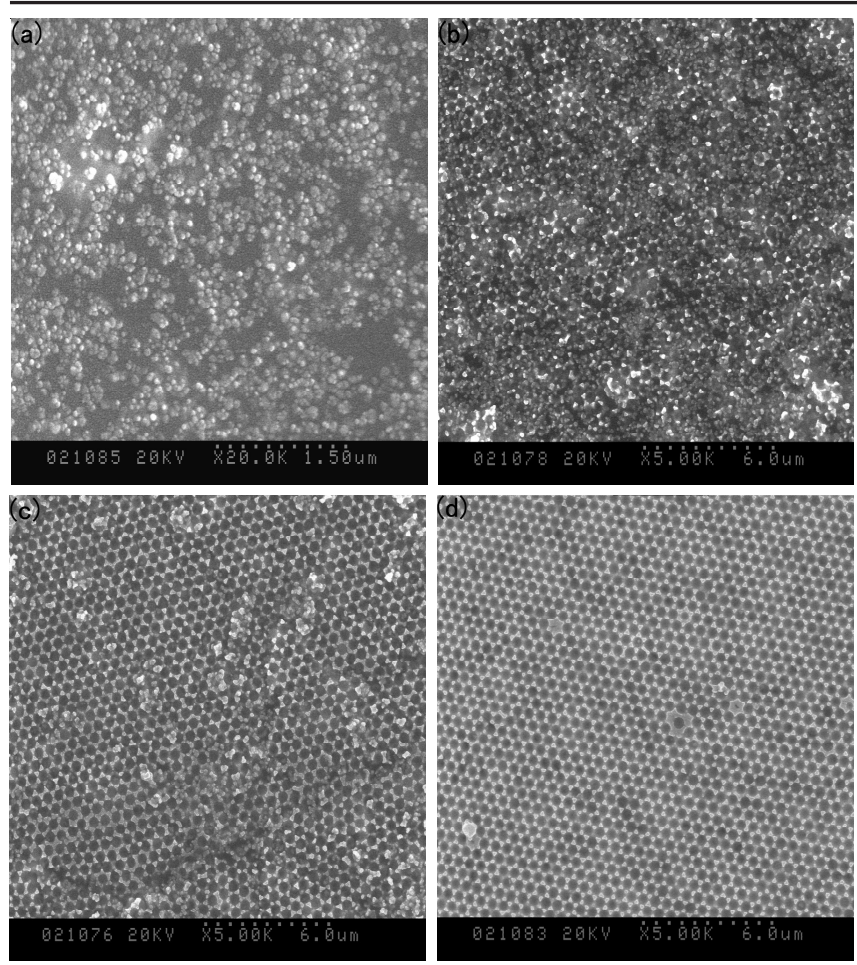

Fig. 3. SEM graphs of silver formed at different deposition time (a) 10 $\min (\mathrm{b}) 30 \mathrm{~min}(\mathrm{c}) 1 \mathrm{~h}(\mathrm{~d}) 2 \mathrm{~h}$

highly ordered metal films with half spherical cavities, this phenomenon results from the jam of first layer nanochannel by Ag nanoparticles, reaction velocity is so rapid that larger silver core in the nanochannel prevent reaction solution more infiltration. At the room temperature, it was happen to stop at the point where the half nanochannel of the first layer of multilayer template. which in turn results in the deposition of a $\mathrm{Ag}$ with a finite thickness covered on the surface. Therefore, more infiltration process is failure when silver core grow up. One hand, the infiltration of reaction solution into polystyrene opal was based on the sample was immersed in the solution container, reactants can permeate more quickly into the opal. In another hand, surface chemistry of the sphere templates influences framework formation, Strong wetting interactions aid pene-tration and formation of a continuous network. Density of silver can be increased by multiple complementary of reactants. It is important that quick reaction and larger core eventually block the penetration of silver amine into bulk of the more than half opal. By such a procedure, Ag grows mostly inside the half layer opal structure.

\section{Conclusion}

We demonstrates a simple and economic approach to design Monolithic metals-Two-dimensional silver nanocavity array was fabricated by templating of the periodically assembled polystyrene nanoparticles via silver mirror reaction and reveal the mechanism of silver growth in the spaceamong the Polystyrene nanoparticle. Polystyrene is made of emulsifierfree emulsion polymerization. Find that silver grow does not depend on specific position on the polystyrene nanoparticle surface, as homogeneous distribution of the Ag core, relying on active surface on Ag core and the left channels, silver grow from silver nanoparticls to two-dimensional silver nanocavity array.

\section{ACKNOWLEDGEMENTS}

This work was supported by the Science Foundation of Jiangsu Marine Resources Development Research Institute (JSIMR11B03).

\section{REFERENCES}

1. P. Jiang, J. Cizeron, J.F. Bertone and V.L. Colvin, J. Am. Chem. Soc., 121, 7957 (1999).

2. X.D. Wang, C.S. Lao, E. Graugnard, C.J. Summers and Z.L. Wang, Nano Lett., 5, 1784 (2005).

3. F. Zeng, Z.W. Sun, C.Y. Wang, B.Y. Ren, X.X. Liu and Z. Tong, Langmuir, 18, 9116 (2002).

4. H.W. Yan, C.F. Blanford, B.T. Holland, M. Parent, W.H. Smyrl and A. Stein, Adv. Mater., 11, 1003 (1999).

5. P.M. Tessier, O.D. Velev, A.T. Kalambur, A.M. Lenhoff, J.F. Rabolt and E.W. Kale, Adv. Mater., 13, 396 (2001).

6. M.C. Netti, S. Coyle, J.J. Baumberg, M.A. Ghanem, P.R. Birkin, P.N. Bartlett and D.M. Whittaker, Adv. Mater., 13, 1368 (2001).

7. C.L. Haynes and R.P. Van Duyne, J. Phys. Chem. B, 105, 5599 (2001).

8. A. Stein and R.C. Schroden, Curr. Opin. Solid State Mater. Sci., 5, 553 (2001).

9. Y. Cui, M.T. Bjo1rk, J.A. Liddle, C. Solnnichsen, B. Boussert and A.P. Alivisatos, Nano Lett., 4, 1093 (2004).

10. S. Coyle, M.C. Netti, J.J. Baumberg, M.A. Ghanem, P.R. Birkin, P.N. Bartlett and D.M. Whittaker, Phys. Rev. Lett., 87, 176801 (2001).

11. P.M. Tessier, O.D. Velev, A.T. Kalambur, J.F. Rabolt, A.M. Lenhoff and E.W. Kaler, J. Am. Chem. Soc., 122, 9554 (2000).

12. P. Jiang, Angew. Chem. Int. Ed., 43, 5625 (2004).

13. J.E.G.J. Wijnhoven, S.J.M. Zevenhuizen, M.A. Hendriks, D. Vanmaekelbergh, J.J. Kelly and W.L. Vos, Adv. Mater., 12, 888 (2000).

14. B.T. Holland, C.F. Blanford, T. Do and A. Stein, Chem. Mater., 11, 795 (1999).

15. D.J. Norris and Y.A. Vlasov, Adv. Mater., 13, 371 (2001).

16. B. Gates, D. Qin and Y. Xia, Adv. Mater., 11, 466 (1999).

17. R. Torrecillas, A. Blanco, M.E. Brito, C. lopez, M. Miguez, F. Meseguer and J.S. Moya, Acta Mater., 48, 4653 ( 2000).

18. Andreas Stein, Micropor. Mesopor. Mater., 44-45, 227 (2001).

19. O.D. Velev and E.W. Kaler, Adv. Mater., 12, 531 (2000). 